\title{
Absorption Ångström coefficient, brown carbon, and aerosols: basic concepts, bulk matter, and spherical particles
}

\author{
H. Moosmüller ${ }^{1}$, R. K. Chakrabarty ${ }^{1}$, K. M. Ehlers ${ }^{2}$, and W. P. Arnott ${ }^{3}$ \\ ${ }^{1}$ Desert Research Institute, Nevada System of Higher Education, Reno, NV 89512, USA \\ ${ }^{2}$ Department of Mathematics, Truckee Meadows Community College, Reno, NV 89512, USA \\ ${ }^{3}$ Department of Physics, University of Nevada, Reno, NV 89557, USA
}

Received: 16 September 2010 - Published in Atmos. Chem. Phys. Discuss.: 22 October 2010

Revised: 29 January 2011 - Accepted: 2 February 2011 - Published: 14 February 2011

\begin{abstract}
The concept of wavelength-dependent absorption Ångström coefficients (AACs) is discussed and clarified for both single and two-wavelengths AACs and guidance for their implementation with noisy absorption spectra is provided. This discussion is followed by application of the concept to models for brown carbon bulk absorption spectra including the damped simple harmonic oscillator model, its Lorentzian approximation, and the band-gap model with and without Urbach tail. We show that the band-gap model with Urbach tail always has an unphysical discontinuity in the first derivative of the AAC at the band-gap - Urbach-tail matching wavelength. Complex refractive indices obtained from the bulk damped simple harmonic oscillator model are used to calculate absorption spectra for spherical particles, followed by a discussion of their features. For bulk material and small particles, this model predicts a monotonic decrease of the AAC with wavelength well above the resonance wavelength; the model predicts a monotonic increase for large particles.
\end{abstract}

\section{Introduction}

The Ångström coefficient was originally introduced as a wavelength-independent constant to describe wavelengthdependent extinction of light by aerosols (Ångström, 1929). It was concluded that it has a "very marked dependence on the size of the scattering particles, but not, in the case of uncoloured substances like $\mathrm{MgO}, \mathrm{ZnO}, \mathrm{SbO}$, on the substance

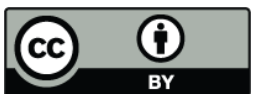

Correspondence to: $\mathrm{H}$. Moosmüller (hansm@dri.edu) itself" (Ångström, 1929). The aerosol size dependence of the Ångström coefficient was known and used to deduce the average diameter of atmospheric aerosols as $\approx 1 \mu \mathrm{m}$ for several surface stations between $35 \mathrm{~m}$ and $2250 \mathrm{~m}$ in altitude. In addition, it was recognized that the ambient Ångström coefficient was far lower during the haziest days of the summer of 1912, after the eruption of Mount Katmai, and it was concluded that the size of volcanic particles from this eruption must have been considerably larger than the ordinary size of dust particles in the atmosphere with an estimated diameter of $\approx 1.5-2.0 \mu \mathrm{m}$ (Ångström, 1929).

In addition to using the Ångström coefficient to describe the wavelength dependence of aerosol scattering or aerosol extinction dominated by scattering, the coefficient has been applied to aerosol and bulk matter absorption spectra. For aerosol absorption dominated by the typical small black carbon combustion particles, the absorption Ångström coefficient AAC remains a wavelength-independent constant, which equals one in the visible and near-visible spectral region (Moosmüller and Arnott, 2009). In this case, the AAC is not dominated by particle size like scattering and highalbedo extinction Ångström coefficients. Recently, multiwavelength photoacoustic instruments have become available (Lewis et al., 2008; Flowers et al., 2010) and initial laboratory and field measurements and related calculations have shown that for many common aerosols, the AAC is not a constant but depends on parameters including wavelength (Lewis et al., 2008; Chakrabarty et al., 2010), particle size (Gyawali et al., 2009), coatings (Lack and Cappa, 2010), chemical composition (Flowers et al., 2010), and possibly morphology (Liu et al., 2008). The use of a variable AAC necessitates further discussion of its definition, determination, and use. In particular, we discuss use of the AAC

Published by Copernicus Publications on behalf of the European Geosciences Union. 
in describing the wavelength-dependent absorption of bulk and particulate brown carbon $(\mathrm{BrC})$ in connection with different bulk theoretical models including the damped simple harmonic oscillator model and its Lorentzian approximation (Demtröder, 2003) and the band-gap model with and without Urbach tail (Sun et al., 2007). The bulk complex refractive index prescribed by the damped simple harmonic oscillator model is used to calculate absorption spectra for spherical particles with a small size parameter approximation (Bohren and Huffman, 1998) and Mie theory (Mie, 1908).

\section{Absorption Ångström coefficients}

The absorption Ångström coefficient AAC is the exponent in a power law expressing the ratio of the absorption coefficients $\alpha(\lambda)$ at two vacuum wavelengths $\lambda_{1}$ and $\lambda_{2}$ as a function of the ratio of these wavelengths as (Moosmüller et al., 2009)

$\frac{\alpha\left(\lambda_{1}\right)}{\alpha\left(\lambda_{2}\right)}=\left(\frac{\lambda_{1}}{\lambda_{2}}\right)^{-\mathrm{AAC}}$.

The AAC can be written explicitly as

$\operatorname{AAC}\left(\lambda_{1}, \lambda_{2}\right)=-\frac{\ln \left(\alpha\left(\lambda_{1}\right)\right)-\ln \left(\alpha\left(\lambda_{2}\right)\right)}{\ln \left(\lambda_{1}\right)-\ln \left(\lambda_{2}\right)}$.

Measurement or calculation of the AAC requires determination of the absorption coefficient $\alpha(\lambda)$ at two wavelengths $\lambda_{1}$ and $\lambda_{2}$ making the AAC specific for this wavelength pair and complicating comparison of different determinations if the AAC is wavelength dependent. Therefore, definition of the $\mathrm{AAC}$ as a function of a single wavelength can be useful.

Figure 1 gives two examples of wavelength dependent absorption coefficients $\alpha(\lambda)$ with $\ln (\alpha)$ (plotted on the y-axis) shown as a function of $\ln (\lambda)$ (plotted on the $\mathrm{x}$-axis). Here the AAC is the negative slope in log-log space. The thin solid line is an example for black carbon (BC) aerosol in the Rayleigh regime, where the size parameter $x$ (i.e., the ratio of particle circumference and wavelengths; see Eq. 11) is much smaller than one and the imaginary part of the refractive index is independent of wavelength, resulting in a straight line with a constant slope of -1 and an AAC of one, independent of wavelength (Bohren and Huffman, 1998; Moosmüller and Arnott, 2009; Moosmüller et al., 2009). The thick solid line is a hypothetical example of what the absorption coefficient of a mixture of $\mathrm{BC}$ and $\mathrm{BrC}$ may look like, that is the sum of a function with the inverse wavelength dependence of $\mathrm{BC}$ and a resonance peak in the UV (in this example, at $0.3 \mu \mathrm{m}$ ) (Andreae and Gelencsér, 2006). Note that for the BrC-BC mixture, the slope and therefore the AAC are dependent on wavelength. For both example absorption spectra, Fig. 1 illustrates measurement of the two-wavelength $\operatorname{AAC}\left(\lambda_{1}, \lambda_{2}\right)$ for $\lambda_{1}=0.4 \mu \mathrm{m}$ and $\lambda_{2}=1.0 \mu \mathrm{m}$, resulting in AACs of 1.0 for $\mathrm{BC}$ and 2.4 for the hypothetical BrC-BC mixture. Obviously for this $\mathrm{BrC}-\mathrm{BC}$ mixture example, measurements for a different wavelength pair would result in a different AAC.

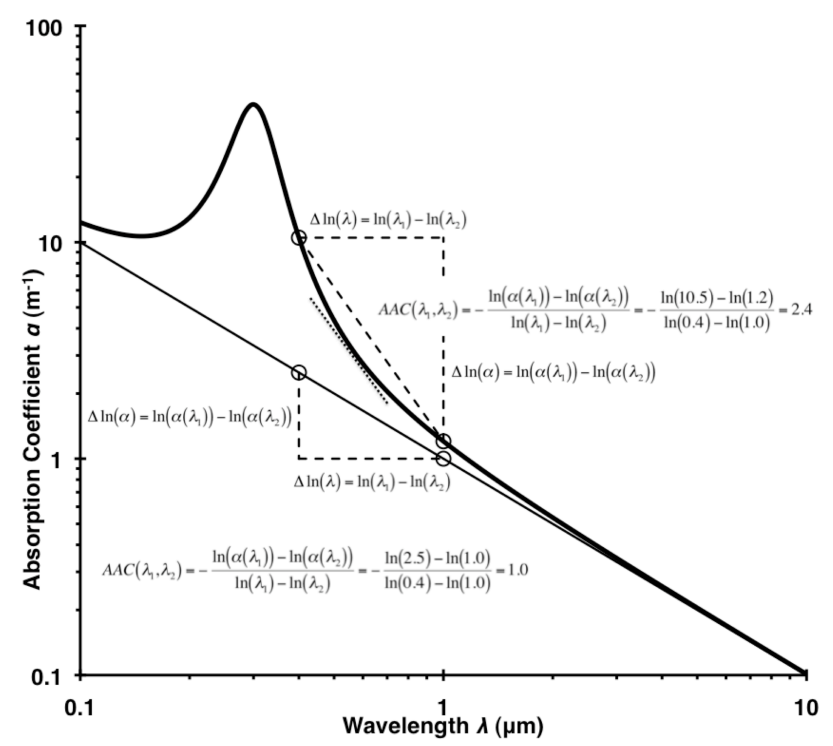

Fig. 1. Illustration of the concept of the absorption Ångström coefficient being the negative slope of the absorption coefficient as a function of wavelength in a log-log plot. The thin solid line is an example for BC aerosol in the Rayleigh regime, while the thick solid line is a hypothetical example of what the absorption coefficient of a mixture of $\mathrm{BC}$ and $\mathrm{BrC}$ may look like, that is the sum of a function with the inverse wavelength dependence of $\mathrm{BC}$ and a resonance peak in the UV.

From this discussion and Fig. 1, the definition of a single wavelength AAC is obvious. It is the negative log-log slope of the absorption spectrum at a wavelength $\lambda$ as shown in Fig. 1 by a dotted line tangential to the absorption spectrum at wavelength $\lambda$. Therefore, the single-wavelength AAC can be written as

$\operatorname{AAC}(\lambda)=-\frac{d \ln (\alpha)}{d \ln (\lambda)}=-\frac{d \ln (\alpha)}{d \lambda} \frac{d \lambda}{d \ln (\lambda)}=-\frac{\lambda}{\alpha(\lambda)} \frac{d \alpha}{d \lambda}$.

Expressions for single-wavelength AACs can be obtained directly from analytical expressions of the absorption coefficient using Eq. (2) or from multi-wavelength measurements with the help of analytical interpolation and/or numerical differentiation.

Single and two-wavelength AACs can be connected by integrating the single-wavelength AAC in $\ln (\lambda)$ space and dividing by the distance in $\ln (\lambda)$ space, thereby yielding the two wavelength Ångström coefficient as

$$
\begin{array}{r}
\operatorname{AAC}\left(\lambda_{1}, \lambda_{2}\right)=\frac{\int_{\lambda_{1}}^{\lambda_{2}} \operatorname{AAC}(\lambda) d \ln (\lambda)}{\ln \left(\lambda_{2}\right)-\ln \left(\lambda_{1}\right)}=\frac{-\int_{\lambda_{1}}^{\lambda_{2}} \frac{\lambda}{\alpha(\lambda)} \frac{d \alpha}{d \lambda} d \ln (\lambda)}{\ln \left(\lambda_{2}\right)-\ln \left(\lambda_{1}\right)} \\
=\frac{-\int_{\lambda_{1}}^{\lambda_{2}} \frac{d \alpha}{\alpha(\lambda)}}{\ln \left(\lambda_{2}\right)-\ln \left(\lambda_{1}\right)}=-\frac{\ln \left(\alpha\left(\lambda_{2}\right)\right)-\ln \left(\alpha\left(\lambda_{1}\right)\right)}{\ln \left(\lambda_{2}\right)-\ln \left(\lambda_{1}\right)}
\end{array}
$$


While the discussion and definitions above are useful for analysis of line shape models, it may be difficult to apply them directly to the analysis of experimental absorption spectra. Experimental data generally contain high-frequency noise and numerical differentiation further increases such noise if directly applied to raw data. However, the AAC concept seeks to describe low-frequency spectral changes. Therefore, noisy spectra first need to be fitted or approximated with theoretical or empirical expressions that reduce high frequency noise and consequently can be used to calculate AACs as discussed above. A simple example is analysis of experimental absorption spectra that have a nearlinear wavelength dependence on a log-log plot between two wavelengths $\lambda_{1}$ and $\lambda_{2}$. Such an analysis can define a twowavelength AAC $\left(\lambda_{1}, \lambda_{2}\right)$ as the negative log-log slope determined by linear regression of the absorption spectrum between the two wavelengths $\lambda_{1}$ and $\lambda_{2}$ (e.g., Hecobian et al., 2010), taking the linear dependence in the log-log plot as an empirical or theoretical model for the wavelength dependence of the absorption coefficient. More sophisticated models have been used for extinction Ångström coefficients, fitting the relationship between aerosol extinction coefficient and wavelength in log-log space with a second-order polynomial (Schuster et al., 2006). This yields an offset, a slope, and a curvature describing the wavelength dependence of extinction, where for zero curvature the negative slope corresponds to the extinction Ångström coefficient.

\section{Brown carbon (BrC) absorption spectra}

We discuss models of $\mathrm{BrC}$ absorption spectra, first for bulk $\mathrm{BrC}$ where the absorption coefficient is directly proportional to the imaginary part of the refractive index. This is followed by use of complex refractive indices calculated with the bulk damped, simple harmonic oscillator model in the approximation of absorption spectra of small $\mathrm{BrC}$ spheres and in Mie theory calculation of absorption spectra of $\mathrm{BrC}$ spheres of arbitrary size.

\subsection{Bulk absorption spectra}

Models for BrC bulk absorption spectra discussed in the following are the damped simple harmonic oscillator model and its Lorentzian approximation plus the band-gap model without and with Urbach tail.

\subsubsection{Damped simple harmonic oscillator model}

Very little is known about the spectrum of the complex refractive index $m(m=n+i k)$ of $\mathrm{BrC}$; therefore, it is described here with a very basic physical expression: the Kramers-Kronig dispersion relations for the damped simple harmonic oscillator (Demtröder, 2003) as
$n_{\mathrm{SHO}}=1+a \frac{v_{0}^{2}-v^{2}}{\left(v_{0}^{2}-v^{2}\right)^{2}+(\gamma v)^{2}}$

and

$k_{\mathrm{SHO}}=a \frac{\gamma v}{\left(v_{0}^{2}-v^{2}\right)^{2}+(\gamma v)^{2}}$,

where $n_{\mathrm{SHO}}$ and $k_{\mathrm{SHO}}$ are the real and imaginary part of the complex refractive index $m_{\mathrm{SHO}}$ for the damped simple harmonic oscillator model, respectively; $v=c / \lambda$ and $v_{0}=c / \lambda_{0}$ are the frequency and resonance frequency of the oscillator, respectively; with $c$ being the speed of light, $a$ is a constant, and $\gamma$ is a line width parameter. An example of the real and imaginary part of the complex refractive index $m_{\mathrm{SHO}}$ is shown in Fig. 2, plotted as a function of wavelength $\lambda$ as solid black lines for $a=10^{29} \mathrm{~s}^{-2}, \gamma=2 \times 10^{14} \mathrm{~s}^{-1}$, and $\lambda_{0}=300 \mathrm{~nm}$ corresponding to $v_{0}=10^{15} \mathrm{~s}^{-1}$. The real part $n_{\mathrm{SHO}}$ has the typical dispersion shape with normal dispersion (i.e., $n$ decreasing with increasing wavelength $\lambda$ for wavelengths well above the resonance wavelength), and a limit of $n_{\mathrm{SHO}}=1+a\left(\lambda_{0} / c\right)^{2}(=1.1$ for the parameters above) for $\lambda \rightarrow \infty$. The imaginary part has the shape of a typical absorption line with its peak at the resonance wavelength $\lambda_{0}$. Note that the assumption of a single resonance frequency is overly simplistic, especially for describing spectral properties in the ultraviolet below the resonance wavelength $\lambda_{0}$, where, for most materials, multiple absorption lines, often resulting in continuous, strong absorption bands are expected. Spectral properties in this region are shown more for an appreciation of the mathematical equations than for a realistic description of spectral properties, and the wavelength region well above the resonance wavelength is most relevant for most applications.

The imaginary part $k$ of the complex refractive index is directly related to the bulk absorption coefficient $\alpha_{\text {bulk }}$ through (Bohren and Huffman, 1998)

$\alpha_{\text {bulk }}=4 \pi \frac{k}{\lambda}$

and $\alpha_{\text {bulk }}$ can be written for the damped simple harmonic oscillator as

$\alpha_{\text {bulk_SHO }}=4 \pi \frac{k_{\mathrm{SHO}}}{\lambda}=\frac{4 \pi a}{c} \frac{\gamma v^{2}}{\left(v_{0}^{2}-v^{2}\right)^{2}+(\gamma \nu)^{2}}$.

The single wavelength AAC for bulk materials $\mathrm{AAC}_{\text {bulk }}$ can be calculated for the damped simple harmonic oscillator model from Eq. (4d) using Eq. (2). Note that for $k$ independent of wavelength (as in the case of $\mathrm{BC}$ ), $\mathrm{AAC}_{\text {bulk }}$ always

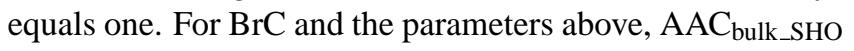
as a function of wavelength $\lambda$ is shown in Fig. 3 as a solid black line. With the $\mathrm{AAC}_{\text {bulk }}$ proportional to the derivative of the imaginary part of the complex refractive index, it has

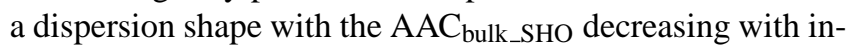
creasing wavelength $\lambda$ for wavelengths well above the resonance wavelength $\lambda_{0}$ towards the limit of $\mathrm{AAC}_{\text {bulk_SHO }}=2$ for $\lambda \rightarrow \infty$. 


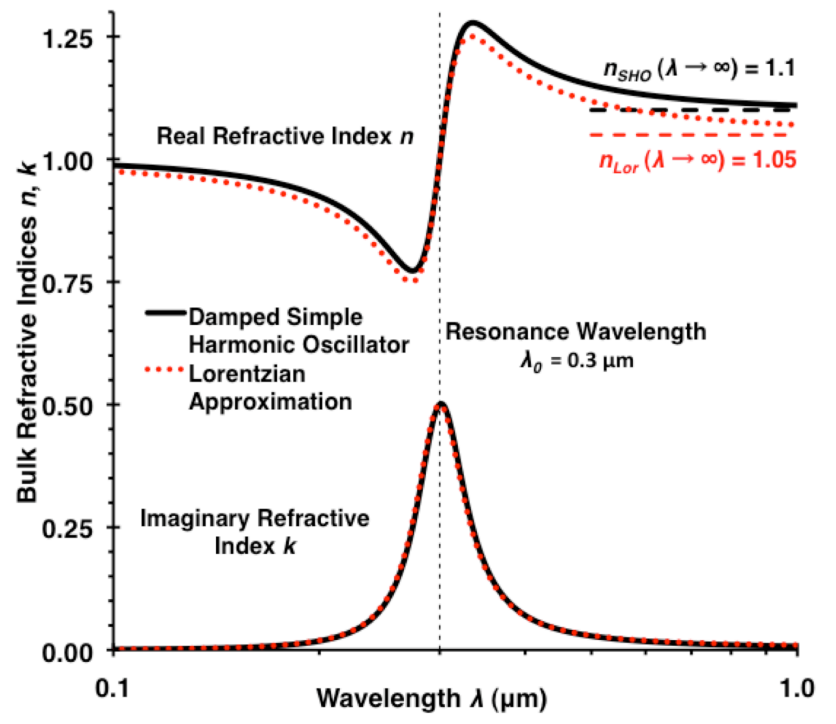

Fig. 2. Real (top lines) and imaginary (bottom lines) refractive indices for the damped simple harmonic oscillator (solid black lines) and its Lorentzian approximation (dotted red lines) as a function of wavelength $\lambda$ (logarithmic scale) with the resonance wavelength $\lambda_{0}=0.3 \mu \mathrm{m}$ shown as vertical dashed line and the long wavelength limits of $n$ as horizontal dashed lines.

\subsubsection{Lorentzian approximation of the damped simple harmonic oscillator model}

Close to the resonance frequency (i.e., $\left|v_{0}-v\right| \ll v_{0}$ ), the term $\left(v_{0}+v\right)$ in the Kramers-Kronig dispersion relations for the damped simple harmonic oscillator (Eqs. 4a, b) can be approximated as

$v+v_{0}=2 v_{0}$,

yielding simplified expressions for the complex refractive index as (Demtröder, 2003)

$n_{\text {Lor }}=1+\frac{a}{2 v_{0}} \frac{v_{0}-v}{\left(v_{0}-v\right)^{2}+(0.5 \gamma)^{2}}$ and

$k_{\text {Lor }}=\frac{a}{4 v_{0}} \frac{\gamma}{\left(v_{0}-v\right)^{2}+(0.5 \gamma)^{2}}$,

where $k_{\text {Lor }}$ has a Lorentzian line shape and both $n_{\text {Lor }}$ and $k_{\text {Lor }}$ are shown in Fig. 2 as dotted red lines for the same parameters used for the curves of the damped simple harmonic oscillator shown in the same figure. Note that the Lorentzian approximation results in sizeable changes for the real part of the complex refractive index away from the resonance wavelength, while the imaginary part is largely unchanged.

The bulk absorption coefficient is proportional to the imaginary part of the complex refractive index (Eq. 4c) and can be written as (Demtröder, 2003)

$\alpha_{\text {bulk_Lor }}=4 \pi \frac{k_{\text {Lor }}}{\lambda}=\frac{\pi a}{c} \frac{\gamma}{\left(\nu_{0}-v\right)^{2}+(0.5 \gamma)^{2}}$.

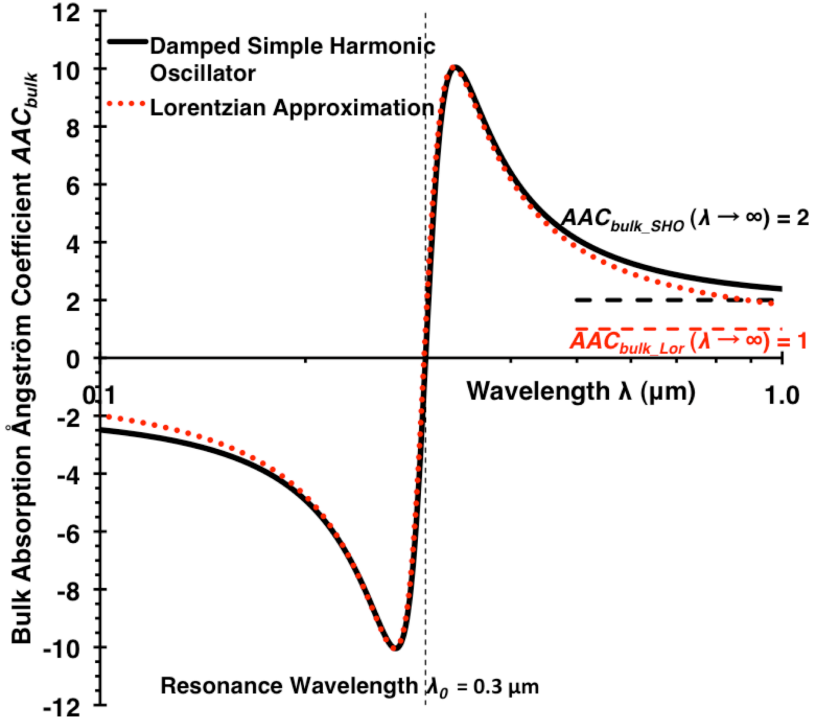

Fig. 3. Bulk absorption Ångström coefficient for the damped simple harmonic oscillator ( $\mathrm{AAC}_{\text {bulk_SHO; }}$; solid black line) and its Lorentzian approximation ( $\mathrm{AAC}_{\text {bulk_Lor }}$; dotted red line) shown as a function of wavelength $\lambda$ (logarithmic scale) with the resonance wavelength $\lambda_{0}=0.3 \mu \mathrm{m}$ shown as the vertical dashed line and the long wavelength limits of $\mathrm{AAC}_{\text {bulk }}$ as horizontal dashed lines.

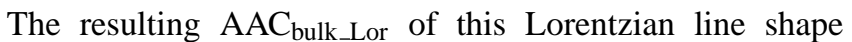
(Eq. 5d) can be calculated with Eq. (2) and is shown in Fig. 3 as a red dotted line. As expected, agreement with the damped simple harmonic oscillator is excellent near the resonance. Away from the resonance, however, $\mathrm{AAC}_{\text {bulk_Lor }}$ has an absolute value substantially smaller than that of $\mathrm{AAC}_{\text {bulk_SHO}}$, and its limit for $\lambda \rightarrow \infty$ is one instead of two. This is due to the fact that in this limit, the assumption for the Lorentzian approximation (i.e., $\left.\left|v_{0}-v\right| \ll v_{0}\right)$ is not fulfilled and results cannot be expected to be in agreement with the damped simple harmonic oscillator model.

\subsubsection{Band-gap model}

The band-gap model (Tauc et al., 1966) has attributed the $\mathrm{AAC}=1$ of $\mathrm{BC}$ to its zero band gap and also has been used to discuss the absorption spectrum of $\mathrm{BrC}$ (Bergstrom et al., 2007; Bond, 2001; Sun et al., 2007). This model relates the bulk absorption coefficient $\alpha_{\text {bulk_BG }}$ to the band-gap energy $E_{\mathrm{BG}}$, the difference between the highest occupied and lowest unoccupied energy state as (Sun et al., 2007)

$$
\begin{aligned}
& \alpha_{\text {bulk_BG }}(\lambda)=C \lambda\left(\frac{1}{\lambda}-\frac{1}{\lambda_{\mathrm{BG}}}\right)^{2}=C\left(\frac{1}{\lambda}-\frac{2}{\lambda_{\mathrm{BG}}}+\frac{\lambda}{\lambda_{\mathrm{BG}}^{2}}\right) \\
& \forall 0 \leq \lambda \leq \lambda_{\mathrm{BG}} \\
& \alpha_{\text {bulk_BG }}(\lambda)=0 \quad \forall \quad \lambda>\lambda_{\mathrm{BG}},
\end{aligned}
$$




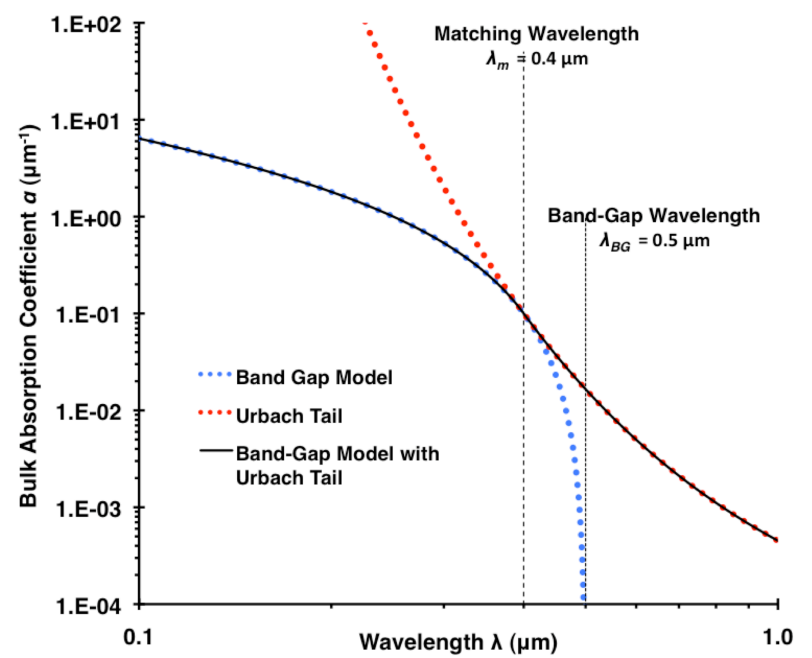

Fig. 4. Bulk absorption coefficient $\alpha$ calculated with the band-gap model (blue dotted line), the Urbach-tail extension (red dotted line), and the band-gap model with Urbach tail (black solid line). Parameters used for this example are $C=1, \lambda_{\mathrm{BG}}=0.5 \mu \mathrm{m}, \lambda_{\mathrm{m}}=0.4 \mu \mathrm{m}$, $\lambda_{0}=3.6 \mu \mathrm{m}$, and $\alpha_{\mathrm{m}}=0.1 \mu \mathrm{m}^{-1}$.

where $\lambda_{\mathrm{BG}}$ is the wavelength corresponding to $E_{\mathrm{BG}}$ (i.e., $\lambda_{\mathrm{BG}}=h c / E_{\mathrm{BG}} ; h$ is Planck's constant), and $C$ is a constant (i.e., $C=B^{2} h c$ in the notation of Sun et al., 2007). Note, however, that some of Sun et al.'s notation is not consistent. For example, with $B$ in the units given in the caption of their Fig. 1, $\alpha$ in their Eq. (5) would have to be replaced by $\alpha / \rho$ as can be shown by simple dimensional analysis. An example of a bulk absorption coefficient $\alpha_{\text {bulk_BG }}$ calculated with the band-gap model (blue dotted line), is shown in Fig. 4 for $C=1$ and $\lambda_{\mathrm{BG}}=0.5 \mu \mathrm{m}$. Note that $\alpha_{\text {bulk_BG }}$ is zero at and above the band-gap wavelength $\lambda_{\mathrm{BG}}$.

Using Eq. (2), the AAC of the band-gap model $\mathrm{AAC}_{\text {bulk_BG }}$ readily can be calculated as

$\operatorname{AAC}_{\text {bulk_BG }}(\lambda)=\frac{\lambda_{\mathrm{BG}}+\lambda}{\lambda_{\mathrm{BG}}-\lambda} \quad \forall \quad 0 \leq \lambda \leq \lambda_{\mathrm{BG}}$

$\operatorname{AAC}_{\text {bulk_BG }}(\lambda)=0 \quad \forall \lambda>\lambda_{\mathrm{BG}}$

in agreement with Sun et al. (2007). An example of $\mathrm{AAC}_{\text {bulk_BG }}$ is shown in Fig. 5 (blue dotted line) for $\lambda_{\mathrm{BG}}=0.5 \mu \mathrm{m}$ as a function of wavelength, demonstrating that $\mathrm{AAC}_{\mathrm{BG}}$ monotonically increases with increasing wavelength, diverging toward $\mathrm{AAC}_{\mathrm{BG}}=\infty$ at $\lambda_{\mathrm{BG}}$. This theoretical behavior is opposite to experimental measurements reporting that $\mathrm{BrC}$ AAC generally decreases with increasing wavelength without showing any divergence at a bandgap wavelength (Russell et al., 2010; Chen and Bond, 2010; Lewis et al., 2008). The drop of the band-gap model absorption coefficient towards the band-gap wavelength, however, is a well-known shortcoming of the simple band-gap model, as displayed in Fig. 5. To mitigate this shortcoming, the band-gap model often is combined with an empirical ex-

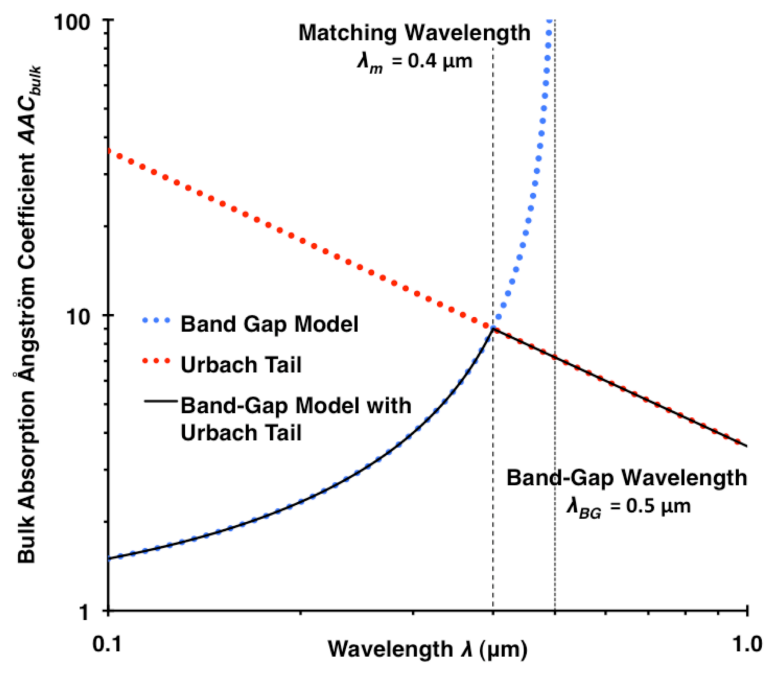

Fig. 5. Bulk absorption Ångström coefficient $\mathrm{AAC}_{\text {bulk calculated }}$ with the band-gap model (blue dotted line), the Urbach-tail extension (red dotted line), and the band-gap model with Urbach tail (black solid line). Parameters used for this example are $\lambda_{\mathrm{BG}}=$ $0.5 \mu \mathrm{m}, \lambda_{\mathrm{m}}=0.4 \mu \mathrm{m}$, and $\lambda_{0}=3.6 \mu \mathrm{m}$.

ponential decay of the absorption coefficient towards longer wavelength, the Urbach tail, which is discussed in the following section.

\subsubsection{Band-gap model with Urbach tail}

Because a few energy states exist within the band gap, absorption spectra exhibit a tail beyond the band-gap wavelength $\lambda_{\mathrm{BG}}$, rather than a sharp cutoff (Sun et al., 2007; Urbach, 1953). This tail can be described with an exponential decay of the absorption coefficient $\alpha_{\text {bulk_Urb }}$ as (Sun et al., 2007)

$\alpha_{\text {bulk_Urb }}(\lambda)=\alpha_{\mathrm{m}} \exp \left(-\lambda_{0}\left(\frac{1}{\lambda_{\mathrm{m}}}-\frac{1}{\lambda}\right)\right)$,

where $\lambda_{0}$ is a characteristic decay width and $\alpha_{\mathrm{m}}=$ $\alpha_{\text {bulk_Urb }}\left(\lambda_{m}\right)$ is the absorption coefficient at a matching wavelength $\lambda_{\mathrm{m}}$. An example of an Urbach-tail absorption coefficient $\alpha_{\text {bulk_Urb }}$ spectrum is shown in Fig. 4 as a red dotted line for $\lambda_{0}=3.6 \mu \mathrm{m}, \lambda_{\mathrm{m}}=0.4 \mu \mathrm{m}$, and $\alpha_{\mathrm{m}}=0.1 \mu \mathrm{m}^{-1}$.

The AAC of the Urbach tail AAC bulk_Urb $_{\text {con be calculated }}$ from Eq. (8) using Eq. (2) as

$\operatorname{AAC}_{\text {bulk_Urb }}(\lambda)=\frac{\lambda_{0}}{\lambda}$.

with limits of $\mathrm{AAC}_{\text {bulk_Urb }}(\lambda=0)=\infty$ and $\mathrm{AAC}_{\text {bulk_Urb }}(\lambda=$ $\infty)=0$. An example of $A_{A C} C_{\text {bulk_Urb }}$ as a function of wavelength is shown in Fig. 5 as a red dotted line for $\lambda_{0}=3.6 \mu \mathrm{m}$.

The Urbach tail can be added to the band-gap model through matching properties of the two models at a matching wavelength $\lambda_{\mathrm{m}}$ below the band-gap wavelength (i.e., 
$\left.\lambda_{\mathrm{m}}<\lambda_{\mathrm{BG}}\right)$. The resulting band-gap/Urbach-tail model uses the band-gap model at wavelengths shorter than the matching wavelength and the Urbach-tail model at longer wavelengths.

Starting this matching process with the absorption coefficient (i.e., $\left.\alpha_{\text {bulk_BG }}\left(\lambda_{\mathrm{m}}\right)=\alpha_{\text {bulk_Urb }}\left(\lambda_{\mathrm{m}}\right)\right)$ gives the parameter $\alpha_{\mathrm{m}}$ in the Urbach-tail model as

$$
\alpha_{\mathrm{m}}=C \lambda_{\mathrm{m}}\left(\frac{1}{\lambda_{\mathrm{m}}}-\frac{1}{\lambda_{\mathrm{BG}}}\right)^{2}=C\left(\frac{1}{\lambda_{\mathrm{m}}}-\frac{2}{\lambda_{\mathrm{BG}}}+\frac{\lambda_{\mathrm{m}}}{\lambda_{\mathrm{BG}}^{2}}\right)
$$

and yields a continuous function for the absorption coefficient $\alpha_{\text {bulk_BG_Urb }}$ in the resulting band-gap/Urbach-tail model as

$$
\begin{aligned}
& \alpha_{\text {bulk_BG_Urb }}(\lambda)=\alpha_{\text {bulk_BG }}(\lambda)=C \lambda\left(\frac{1}{\lambda}-\frac{1}{\lambda_{\text {BG }}}\right)^{2} \\
& \forall \quad \lambda \leq \lambda_{\mathrm{m}} \\
& \alpha_{\text {bulk_BG_Urb }}(\lambda)=\alpha_{\text {bulk_Urb }}(\lambda) \\
& =C \lambda_{\mathrm{m}}\left(\frac{1}{\lambda_{\mathrm{m}}}-\frac{1}{\lambda_{\mathrm{BG}}}\right)^{2} \exp \left(-\lambda_{0}\left(\frac{1}{\lambda_{\mathrm{m}}}-\frac{1}{\lambda}\right)\right) \forall \lambda \geq \lambda_{\mathrm{m}} .
\end{aligned}
$$

In addition to obtaining a continuous function for the absorption coefficient, it also is desirable to obtain a continuous function for the AAC of the resulting band-gap/Urbach-tail model $\mathrm{AAC}_{\text {bulk_BG_Urb}}$, which is equivalent to a continuous first derivative of the absorption coefficient. This match gives the characteristic decay width $\lambda_{0}$ of the Urbach model as

$\lambda_{0}=\lambda_{\mathrm{m}} \frac{\lambda_{\mathrm{BG}}+\lambda_{\mathrm{m}}}{\lambda_{\mathrm{BG}}-\lambda_{\mathrm{m}}}$,

for use in Eq. (10b).

Unfortunately, additional matching of the first derivatives of the AACs cannot be achieved. Therefore, the first deriva-

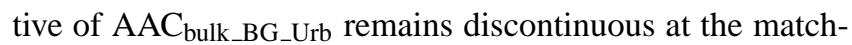
ing wavelength. An example of this can be seen in Fig. 5,

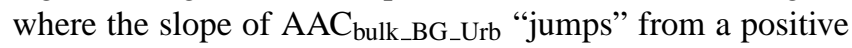
to a negative value at the matching wavelength. Obviously, this kind of behavior is unphysical and has, to the best of our knowledge, not been observed. Therefore, even the addition of the Urbach tail to the band-gap model does not yield a sensible model of $\mathrm{AAC}_{\text {bulk }}$ in agreement with observations, making the band-gap/Urbach-tail model unsuitable for explaining the behavior of the bulk BrC AAC.

\subsection{Absorption spectra of spherical brown carbon (BrC) particles}

Absorption spectra of particles depend on the properties of the bulk matter, particle morphology, and size (Moosmüller et al., 2009). Here, we limit discussion of particle absorption spectra to those of homogeneous, spherical particles as a function of particle diameter.

A complete analytical solution of Maxwell's equations for the scattering of infinite electromagnetic plane waves from homogeneous, linear, isotropic, spherical particles (Bohren and Huffman, 1998; Mie, 1908) has been developed by the German physicist Gustav Mie (Lilienfeld, 1991; Mishchenko and Travis, 2008) and is commonly known as Mie theory. This theory calculates particle optical properties from the complex refractive index of the particle and that of its surrounding medium (here assumed to be 1) and the size parameter $x$ of the particle, which is the ratio of particle circumference $\pi d$ ( $d$ being the particle diameter) and wavelength $\lambda$ of the electromagnetic wave as

$x=\frac{\pi d}{\lambda}$.

Mie theory uses bulk material properties contained in the complex refractive index spectrum to obtain the absorption spectra of particles. If the complex refractive index is independent of wavelength $\lambda$ (such as for BC in the visible and near-visible spectrum), Mie theory is scale invariant and depends only on the size parameter $x$ (Mishchenko, 2006). For an explicit wavelength dependence of the complex refractive index (such as for $\mathrm{BrC}$ ), particle size and electromagnetic wavelength need to be considered separately.

While Mie theory contains solutions for all particle size parameters, it is often useful to investigate simpler solutions for specific size parameter ranges to gain additional physical insight. This is done in the following section for small spherical particles, followed by a section discussing Mie calculations for a large range of particle sizes.

\subsubsection{Small spherical particles}

For small spherical particles, that is for particles with a size parameter $x \ll 1$, the absorption cross-section $\sigma_{\text {abs }}$ can be written as (Bohren and Huffman, 1998; Sun et al., 2007)

$\sigma_{\mathrm{abs}}=\frac{6 \pi V}{\lambda} \operatorname{Im}\left(\frac{m^{2}-1}{m^{2}+2}\right)=\alpha_{\text {bulk }} V \xi(\lambda)$

where Im denotes the imaginary part of its argument; and the absorption cross-section for small spherical particles is the product of their volume $V$, bulk absorption coefficient $\alpha_{\text {bulk, }}$, and of $\xi(\lambda)$, a weak function of the imaginary refractive index $k$, with (Sun et al., 2007)

$\xi(\lambda)=\frac{9 n}{\left(n^{2}-k^{2}+2\right)^{2}+4 n^{2} k^{2}}$.

This function is shown in Fig. 6 for the complex refractive index $m=n+i k$ of the damped simple harmonic oscillator model specified in Eq. (4) with $a=10^{29} \mathrm{~s}^{-2}, \gamma=$ $2 \times 10^{14} \mathrm{~s}^{-1}$, and $\lambda_{0}=300 \mathrm{~nm}$ as used in the discussion of bulk matter absorption. Note that $\xi$ has a dispersion shape and in this example depends only weakly $(<15 \%$ deviation from one) on wavelength, changing only by a few percent in the visible spectral region. Therefore, the absorption cross-sections of these small spherical particles have a wavelength dependence very similar to that of the bulk 


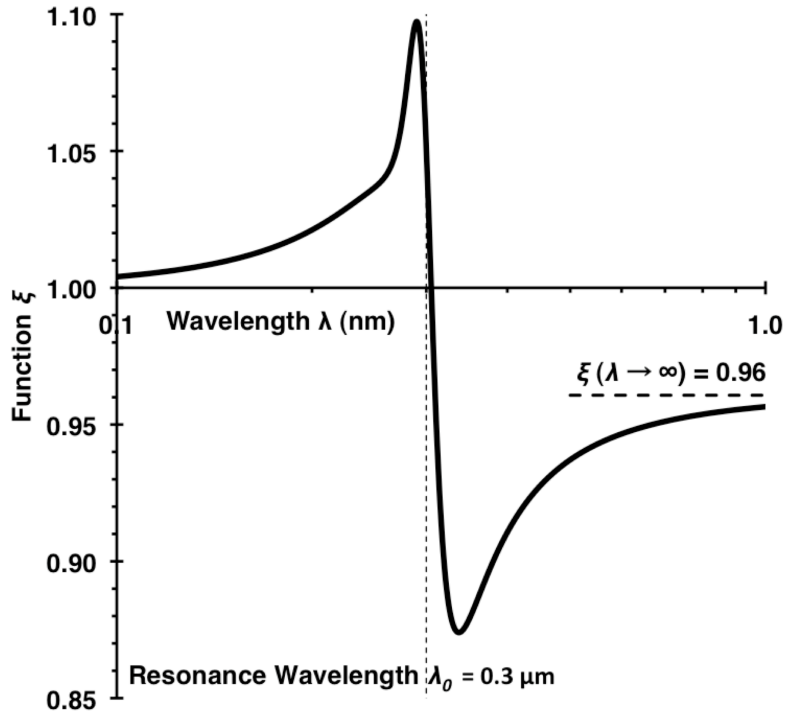

Fig. 6. Function $\xi$ for the complex refractive index $m=n+i k$ specified in Eq. (4) as a function of wavelength $\lambda$ (logarithmic scale) with the resonance wavelength $\lambda_{0}=0.3 \mu \mathrm{m}$ shown as a vertical dashed line and the long wavelength limit of $\xi$ as a horizontal dashed line. Note that $\xi$ depends only weakly on the wavelength resulting in absorption cross-sections for small spherical particles that have wavelength dependence very similar to that of the bulk absorption coefficient.

absorption coefficient. The long wavelength limit of $\xi$ is $\xi_{\infty}=9 n_{\infty} /\left(n_{\infty}^{2}+2\right)^{2}$ with $n_{\infty}=1+a\left(\lambda_{0} / c\right)^{2}$ for $\lambda \rightarrow \infty$. We compare the resulting mass absorption efficiency $E_{\text {abs }}$ and AAC spectra of small spherical particles with those from Mie theory and of bulk matter in Figs. 7 and 8, demonstrating agreement with small particle (i.e., $d=0.01 \mu \mathrm{m}$ ) Mie theory and similarity and a small enhancement of the AAC of small particles compared to that of bulk matter near the resonance frequency.

\subsubsection{Spherical particles of arbitrary size}

For homogeneous, spherical particles with a diameter ranging from 0.01 to $10 \mu \mathrm{m}$, Mie theory has been used to calculate their mass absorption efficiency as a function of wavelength $\lambda$ ranging from 0.01 to $10 \mu \mathrm{m}$. The mass absorption efficiency $E_{\text {abs }}$ is defined as the particle absorption cross section $\sigma_{\text {abs }}$ per particle mass $m$ or

$E_{\mathrm{abs}}=\frac{\sigma_{\mathrm{abs}}}{m}=\frac{\sigma_{\mathrm{abs}}}{\rho V}=\frac{6 \sigma_{\mathrm{abs}}}{\rho \pi d^{3}}$,

where $\rho$ and $V$ are particle mass density and particle volume, respectively. Results of these Mie theory calculations are shown in Fig. 7 with $\rho=1 \mathrm{~g} \mathrm{~cm}^{-3}$ for the damped simple harmonic oscillator and parameters identical to those used for previous figures together with results for the small spher-

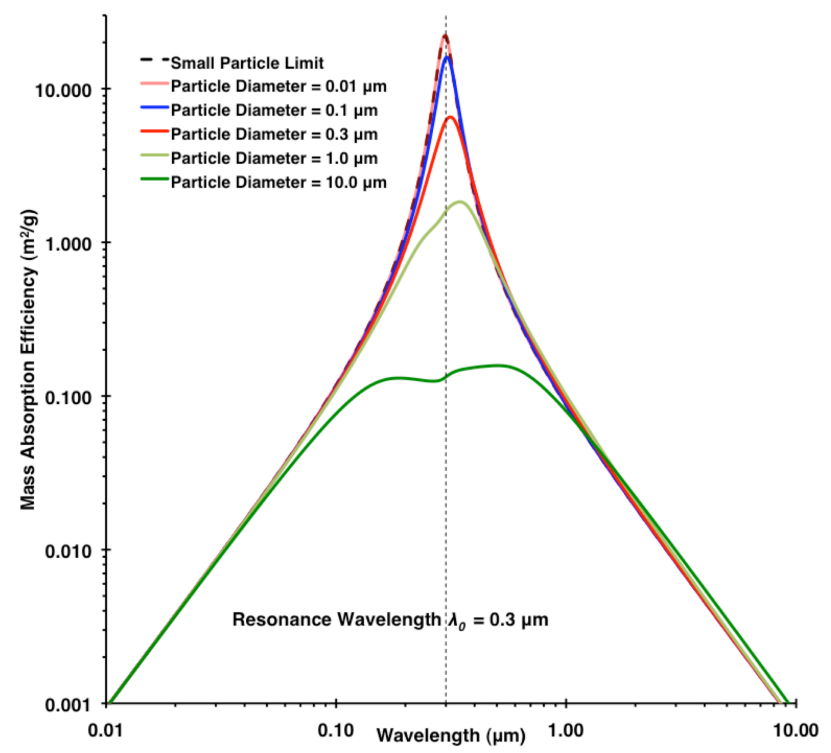

Fig. 7. Damped simple harmonic oscillator model wavelength dependence of the mass absorption efficiency ( $E_{\mathrm{abs}}$; for a mass density of $1 \mathrm{~g} \mathrm{~cm}^{-3}$ ) for fine particles (Eq. 12 and Fig. 6) and for spherical particles with particle diameter ranging from $0.01 \mu \mathrm{m}$ to $10 \mu \mathrm{m}$ from Mie theory calculations using the bulk refractive index (Eqs. 4a, b and Fig. 2).

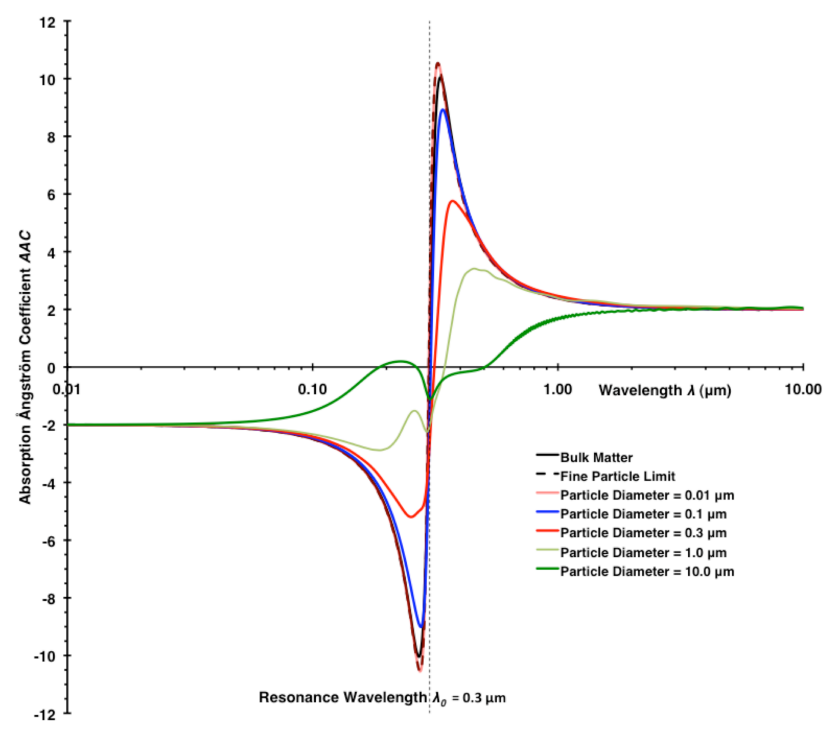

Fig. 8. Damped simple harmonic oscillator model wavelength dependence of the absorption Ångström coefficient (AAC) for bulk matter (Eqs. 4b, c and Fig. 3), fine particles (Eq. 12), and spherical particles with particle diameter ranging from $0.01 \mu \mathrm{m}$ to $10 \mu \mathrm{m}$ from Mie theory calculations using the bulk refractive index (Eqs. 4a, b).

ical particle approximation. Interpretation of this figure depends upon the spectra of the complex refractive index and absorption properties of spherical particles. For the shortest wavelength shown (i.e., $\lambda=0.01 \mu \mathrm{m}$ ), the imaginary part 
of the refractive index is very small (i.e., $k=7.4 \times 10^{-7}$ ) corresponding to a bulk absorption coefficient of $\alpha_{\text {bulk }}=$ $9.4 \times 10^{-4} \mu \mathrm{m}^{-1}$, which allows the light to fully penetrate virtually all particle sizes, resulting in volume absorbers with nearly identical mass absorption efficiency (Sun et al., 2007) as obvious in Fig. 7. For the longest wavelength shown (i.e., $\lambda=10 \mu \mathrm{m}$ ), the imaginary part of the refractive index is not as small (i.e., $k=6.0 \times 10^{-4}$ ) as for the shortest wavelength, however the longer wavelength results in a comparable bulk absorption coefficient of $\alpha_{\text {bulk }}=7.6 \times 10^{-4} \mu \mathrm{m}^{-1}$. Therefore, full penetration of the spheres by incident light is expected. As a particle size parameter $x$ of about one is approached, however, particle absorption is enhanced by internal resonances yielding increased mass absorption efficiencies (i.e., by $\approx 15 \%$ ) for the largest particle size (i.e., $10 \mu \mathrm{m}$ in diameter), as clearly visible in Fig. 7.

The spectra for the small particle limit (Eq. 12) and for the particle diameter of $0.01 \mu \mathrm{m}$ show excellent agreement with each other and peak just slightly below the resonance wavelength (i.e., $\lambda_{0}=0.3 \mu \mathrm{m}$ ), where the bulk absorption coefficient and imaginary part of the refractive index peak. This slight shift is related to the asymmetry of the function $\xi(\lambda)$ (see Eq. 12; Fig. 6) and the real part of the refractive index $n(\lambda)$ (see Eq. 4; Fig. 4). Toward larger particle diameters, the peak of the mass absorption efficiency is lowered and broadened due to incomplete light penetration of the particle, where a decreasing particle volume fraction contributes to the absorption. In addition, the peak is shifted above the resonance wavelength, where in the geometric optics interpretation $(x \gg 1)$, the Fresnel reflectivity is reduced and more light penetrates the sphere to be absorbed (Moosmüller and Arnott, 2009).

Using Eq. (2), the AAC spectra of these spherical particles can be calculated readily from the absorption coefficient spectra used for Fig. 7. Results are shown in Fig. 8 together with the AAC for bulk matter. Interpretation of these AAC spectra is closely related to interpretation of the mass absorption efficiency $E_{\text {abs }}$ spectra in Fig. 7 discussed above and focuses on the region above the resonance wavelength that is of most practical importance. Spectra for the particle diameter of $0.01 \mu \mathrm{m}$ are in excellent agreement with those for the fine particle limit discussed in the previous section (Small Spherical Particles). With increasing particle size, the AAC peak above the resonance wavelength is lowered, broadened, and shifted toward longer wavelengths until it disappears for a particle diameter between 1 and $10 \mu \mathrm{m}$ and is replaced by a monotonic increase of AAC above the resonance wavelength as seen for a particle diameter of $10 \mu \mathrm{m}$. We are not aware of any experimental determination of the AAC spectrum for $\mathrm{BrC}$ particles in this size range.

\section{Conclusions}

The concept of wavelength-dependent AACs has been clarified including the description and mathematical connection of single- and two-wavelength AACs among each other and to absorption spectra. These concepts can be applied to noisy experimental and theoretical data by utilizing theoretical or empirical spectral models. We applied this discussion of wavelength-dependent AACs to models of $\mathrm{BrC}$ bulk absorption spectra including the damped simple harmonic oscillator model, its Lorentzian approximation, and the band-gap model with and without Urbach tail. The simple harmonic oscillator model yields bulk AAC spectra that are physically reasonable and in qualitative agreement with experimental observations and yield $\mathrm{AACs} \geq 2$, well above the resonance wavelength. The band-gap model without Urbach tail yields AACs monotonically increasing with wavelength, in qualitative disagreement with experimental observations, and diverging toward infinity at the band-gap wavelength. While the addition of an Urbach tail truncates this divergence, yielding a monotonic increase of AAC below and a monotonic decrease above the matching wavelength between band-gap and Urbach-tail models. This instantaneous change in AAC slope corresponds to an unphysical discontinuity of the first derivative of the AAC spectrum at the matching wavelength and makes the use of the band-gap model with and without Urbach tail questionable for the description of BrC AAC spectra.

The use of complex refractive indices obtained from the bulk damped simple harmonic oscillator model for calculation of absorption and $\mathrm{AAC}$ spectra for spherical $\mathrm{BrC}$ particles results in prediction of a monotonic decrease of the AAC with wavelengths well above the resonance wavelength for small particles but of a monotonic increase for large particles. While the decrease for small particles is commonly observed, we are not aware of corresponding observations for large particles.

These discussions clearly demonstrate the utility of the $\mathrm{AAC}$ for wavelength dependent imaginary refractive indices as the AAC helps to distinguish absorption spectra of $\mathrm{BC}$ particles $(\mathrm{AAC}=1)$ from $\mathrm{BrC}$ particles $(\mathrm{AAC} \gg 1)$ and helps to evaluate spectral models such as the band-gap model with and without Urbach tail and the damped simple harmonic oscillator model. Laboratory evaluations of different theoretical absorption lineshape models are clearly needed and should commence with measuring absorption spectra of UV-absorbing organic substances for bulk material and for monodisperse spheres of different sizes. Further work is needed in extending theoretical description and experimental verification to non-spherical and inhomogeneous particles such as a BC core coated with a BrC shell and fractal-like BC particles coated with $\mathrm{BrC}$ and to externally mixed particles. In addition, a true understanding of the absorption spectra of $\mathrm{BrC}$ aerosols will depend on identifying the OC components or groups of components that cause the increased light 
absorption at shorter wavelengths, to quantify their absorption spectra, and to thereby relate the purely optical definition of $\mathrm{BrC}$ to its chemistry.

Acknowledgements. This material is based upon work supported by NASA EPSCoR under Cooperative Agreement No. NNX10AR89A, by NASA ROSES under Grant NNX11AB79G, by the National Science Foundation under Cooperative Support Agreement No. EPS-0814372 and under Major Research Instrumentation Grant AGS-1040046, and by the Desert Research Institute.

\section{Edited by: V. F. McNeill}

\section{References}

Andreae, M. O. and Gelencsér, A.: Black carbon or brown carbon? The nature of light-absorbing carbonaceous aerosols, Atmos. Chem. Phys., 6, 3131-3148, doi:10.5194/acp-6-3131-2006, 2006.

Ångström, A.: On the Atmospheric Transmission of Sun Radiation and on Dust in the Air, Geogr. Ann., 11, 156-166, 1929.

Bergstrom, R. W., Pilewskie, P., Russell, P. B., Redemann, J., Bond, T. C., Quinn, P. K., and Sierau, B.: Spectral absorption properties of atmospheric aerosols, Atmos. Chem. Phys., 7, 5937-5943, doi:10.5194/acp-7-5937-2007, 2007.

Bohren, C. F. and Huffman, D. R.: Absorption and Scattering of Light by Small Particles, Wiley, New York, xiv, 530 pp., 1998.

Bond, T. C.: Spectral Dependence of Visible Light Absorption by Carbonaceous Particles Emitted from Coal Combustion, Geophys. Res. Lett., 28, 4075-4078, 2001.

Chakrabarty, R. K., Moosmüller, H., Chen, L.-W. A., Lewis, K., Arnott, W. P., Mazzoleni, C., Dubey, M. K., Wold, C. E., Hao, W. M., and Kreidenweis, S. M.: Brown carbon in tar balls from smoldering biomass combustion, Atmos. Chem. Phys., 10, 6363-6370, doi:10.5194/acp-10-6363-2010, 2010.

Chen, Y. and Bond, T. C.: Light absorption by organic carbon from wood combustion, Atmos. Chem. Phys., 10, 1773-1787, doi:10.5194/acp-10-1773-2010, 2010.

Demtröder, W.: Laser Spectroscopy: Basic Concepts and Instrumentation, 3 ed., Springer Verlag, Berlin, Heidelberg, New York, 380 pp., 2003.

Flowers, B. A., Dubey, M. K., Mazzoleni, C., Stone, E. A., Schauer, J. J., Kim, S.-W., and Yoon, S. C.: Optical-chemicalmicrophysical relationships and closure studies for mixed carbonaceous aerosols observed at Jeju Island; 3-laser photoacoustic spectrometer, particle sizing, and filter analysis, Atmos. Chem. Phys., 10, 10387-10398, doi:10.5194/acp-10-103872010, 2010.

Gyawali, M., Arnott, W. P., Lewis, K., and Moosmüller, H.: In situ aerosol optics in Reno, NV, USA during and after the summer 2008 California wildfires and the influence of absorbing and non-absorbing organic coatings on spectral light absorption, Atmos. Chem. Phys., 9, 8007-8015, doi:10.5194/acp-9-8007-2009, 2009.
Hecobian, A., Zhang, X., Zheng, M., Frank, N., Edgerton, E. S., and Weber, R. J.: Water-Soluble Organic Aerosol material and the light-absorption characteristics of aqueous extracts measured over the Southeastern United States, Atmos. Chem. Phys., 10, 5965-5977, doi:10.5194/acp-10-5965-2010, 2010.

Lack, D. A. and Cappa, C. D.: Impact of brown and clear carbon on light absorption enhancement, single scatter albedo and absorption wavelength dependence of black carbon, Atmos. Chem. Phys., 10, 4207-4220, doi:10.5194/acp-10-4207-2010, 2010.

Lewis, K., Arnott, W. P., Moosmüller, H., and Wold, C. E.: Strong Spectral Variation of Biomass Smoke Light Absorption and Single Scattering Albedo Observed with a Novel DualWavelength Photoacoustic Instrument, J. Geophys. Res., 113, D16203, doi:10.1029/2007JD009699, 2008.

Lilienfeld, P.: Gustav Mie: The Person, Appl. Opt., 30, 4696-4698, 1991.

Liu, L., Mishchenko, M. I., and Arnott, W. P.: A Study of Radiative Properties of Fractal Soot Aggregates Using the Superposition TMatrix Method, J. Quant. Spectrosc. Ra., 109, 2656-2663, 2008.

Mie, G.: Beiträge zur Optik trüber Medien, speziell kolloidaler Metallösungen, Ann. Physik, 330, 377-445, 1908.

Mishchenko, M. I.: Scale Invariance Rule in Electromagnetic Scattering, J. Quant. Spectrosc. Ra., 101, 411-415, 2006.

Mishchenko, M. I. and Travis, L. D.: Gustav Mie and the Evolving Discipline of Electromagnetic Scattering by Particles, B. Am. Meteor. Soc., 89, 1853-1861, 2008.

Moosmüller, H. and Arnott, W. P.: Particle Optics in the Rayleigh Regime, J. Air Waste Manage. Assoc., 59, 1028-1031, 2009.

Moosmüller, H., Chakrabarty, R. K., and Arnott, W. P.: Aerosol Light Absorption and its Measurement: A Review, J. Quant. Spectrosc. Ra., 110, 844-878, 2009.

Russell, P. B., Bergstrom, R. W., Shinozuka, Y., Clarke, A. D., DeCarlo, P. F., Jimenez, J. L., Livingston, J. M., Redemann, J., Dubovik, O., and Strawa, A.: Absorption Angstrom Exponent in AERONET and related data as an indicator of aerosol composition, Atmos. Chem. Phys., 10, 1155-1169, doi:10.5194/acp10-1155-2010, 2010.

Schuster, G. L., Dubovik, O., and Holben, B. N.: Angstrom Exponent and Bimodal Aerosol Size Distributions, J. Geophys. Res., 111, D07207, doi:10.1029/2005JD006328, 2006.

Sun, H. L., Biedermann, L., and Bond, T. C.: Color of Brown Carbon: A Model for Ultraviolet and Visible Light Absorption by Organic Carbon Aerosol, Geophys. Res. Lett., 34, L17813, doi:10.1029/2007GL029797, 2007.

Tauc, J., Grigorovici, R., and Vancu, A.: Optical Properties and Electronic Structure of Amorphous Germanium, Phys. Stat. Sol., 15, 627-637, 1966.

Urbach, F.: The Long-Wavelength Edge of Photographic Sensitivity and of the Electronic Absorption of Solids, Phys. Rev., 92, p. $1324,1953$. 